\title{
Statistical Characteristics of Measured 3-Dimensional MIMO Channel for Outdoor-to-Indoor Scenario in China and New Zealand
}

\author{
Yawei Yu, ${ }^{1}$ Jianhua Zhang, ${ }^{1}$ Mansoor Shafi, ${ }^{2}$ Min Zhang, ${ }^{3}$ and Jawad Mirza ${ }^{4}$ \\ ${ }^{1}$ Beijing University of Posts and Telecommunications, Beijing 100876, China \\ ${ }^{2}$ Spark New Zealand, Wellington 6011, New Zealand \\ ${ }^{3}$ Alcatel-Lucent, Swindon SN5 7DJ, UK \\ ${ }^{4}$ Victoria University of Wellington, Wellington 6012, New Zealand \\ Correspondence should be addressed to Yawei Yu; yyw@bupt.edu.cn
}

Received 8 October 2015; Revised 15 January 2016; Accepted 8 February 2016

Academic Editor: Enrico Del Re

Copyright (c) 2016 Yawei Yu et al. This is an open access article distributed under the Creative Commons Attribution License, which permits unrestricted use, distribution, and reproduction in any medium, provided the original work is properly cited.

\begin{abstract}
The 3-dimensional (3D) channel model gives a better understanding of statistical characteristics for practical channels than the 2-dimensional (2D) channel model, by taking the elevation domain into consideration. As different organizations and researchers have agreed to a standard 3D channel model, we attempt to measure the 3D channel and determine the parameters of the standard model. In this paper, we present the statistical propagation results of the 3D multiple-input and multiple-output (MIMO) channel measurement campaign performed in China and New Zealand (NZ). The measurements are done for an outdoor-to-indoor (O2I) urban scenario. The dense indoor terminals at different floors in a building form a typical 3D propagation environment. The key parameters of the channel are estimated from the measured channel impulse response (CIR) using the spatial-alternating generalized expectation-maximization (SAGE) algorithm. Till now there is abundant research performed on the azimuth domain; this paper mainly considers the statistical characteristics of the elevation domain. A statistical analysis of 3D MIMO channel results for both China and NZ measurements is presented for the following parameters: power delay profile (PDP), root mean square (rms), delay spread (DS), elevation angle-of-arrival (EAoA) distribution, elevation angle-of-departure (EAoD) distribution, elevation angular spread (AS), and cross-polarization discrimination (XPD).
\end{abstract}

\section{Introduction}

The mobile traffic is experiencing an explosive growth due to the flourishing spread of mobile internet and smart phones. It is predicted that the mobile traffic will grow more than 1000 times in the next few years [1]. To fulfill this increasing capacity demand there have been a lot of research activities under the umbrella of $5 \mathrm{G}$ mobile communication system [2]. Among all of these activities, one important topic is the 3-dimensional (3D) channel model, which, in addition to the azimuth domain, also takes the elevation domain into consideration, thus providing additional degrees of freedom to meet the high capacity demand [3].

Conventional multiple-input and multiple-output (MIMO) technology only utilizes the horizontal plane of the spatial domain in the spatial signal processing. Usually, a linear antenna array with vertical polarization or cross-polarization is deployed in the horizontal plane at both ends of the communication link, while a fixed downtilting angle is configured at the antenna array of the base station (BS). Although the signal propagates through a $3 \mathrm{D}$ space, the fading channel is still modeled as 2-dimensional (2D) channel models. With the advent of the adaptive antenna array, the realization of $3 \mathrm{D}$ MIMO is more practical. Thus an extensive interest has been motivated to exploit the elevation domain in 3D MIMO system [4].

An early research on the elevation angle can be traced back to 1979 by Aulin, who extended Clark's scattering model to 3D space for the first time [5]. Since then, a growing number of researchers observed and reported the significance of 
elevation angles in describing the radio signal propagation more precisely. The impact of elevation angle on MIMO capacity was presented in [6] and vertical sectorization was described in [7]. Simulation results show that 3D MIMO system with 32 antenna ports achieves 2 3.6 times cell average throughput gain compared to the $4 \mathrm{G}$ LTE system of two antenna ports at the BS [8]. Further practical field measurements have validated the superiority of 3D MIMO channel by harvesting a $27 \% \sim 42 \%$ capacity gain in UMi scenario [9], which highlights the importance of elevation domain. 3D characteristics, including the elevation distribution, mean elevation angle (MEA) and elevation angle spread (EAS), are analyzed based on field channel measurements in [10-14]. The Third Generation Partner Project (3GPP) has initiated the discussion on the general 3D channel fading model $[15,16]$ since January 2013 and released the 3D MIMO channel model [17] in April 2014. World Wireless Initiative New Radio + (WINNER+) has also reported 3D channel measurements [18] as an extension of the 2D geometry based stochastic model (GBSM). A good survey of 3D channel models is given in [19]. Six proposals to the 3GPP based on our measurements and analysis are presented in [20-25]. Reliable and realistic channel models based on the empirical propagation characteristics are critical to fundamental research and evaluation of the 3D MIMO technique. However, the understanding of 3D MIMO channel is still in its infancy; how to characterize $3 \mathrm{D}$ propagation channels, especially the elevation angle, is therefore an important task for 3D MIMO development, yet practical measurements of the 3D fading channel are scarce. As the standard organizations and channel modeling researchers have agreed to a standard 3D channel model, it is our attempt to measure the channel and determine its parameters according to the standard model, then model researchers can generate the wireless channel impulse response (CIR) for system simulation studies.

In this paper, we present efforts to the study of the statistical characteristics of the 3D MIMO channel for outdoorto-indoor (O2I) scenario. Using the same measurement equipment, measurements are repeated for two locations with very diverse surroundings in China and NZ, respectively. This helps to compare the similarities and differences between the results. We report on measurements of a 3D MIMO CIR and give results for the following channel parameters:

(i) power delay profile (PDP),

(ii) distribution of the delay spread (DS),

(iii) distributions of elevation angle-of-arrival (EAoA) and elevation angle-of-departure (EAoD),

(iv) distribution of the angle spread (AS) in the elevation domain,

(v) cross-polarization discrimination (XPD).

In Section 2, detailed descriptions for the measurement scenario and data postprocessing will be presented, followed by measurement results of key parameters for the 3D MIMO channel in Section 3 and conclusions in Section 4.

\section{Measurement Description}

2.1. Measurement Equipment and Scenario Description. Measurements are done by using the Elektrobit Propsound Sounder described in [26]. It consists of three units: transmitter $(\mathrm{Tx})$ sounder, receiver $(\mathrm{Rx})$ sounder, and $\mathrm{Rx}$ Baseband Processing Unit (RBPU), through which signals can be transmitted, received, and stored in a time division way, respectively.

To capture the propagation rays in the $3 \mathrm{D}$ environment efficiently, fully dimensional antenna arrays were equipped at both sides of the measurement link. The layout of the antenna arrays at Tx and Rx side are illustrated in Figures 1(a) and 1(b), respectively. At the $\mathrm{Rx}$, dual-polarized omnidirectional array (ODA) consisting of 56 antenna elements with 8 adjacent sides and a top surface was used, while a dual-polarized uniform planar array (UPA) with 32 antenna elements was utilized as a sector transmit antenna array. All array elements consisted of microstrip patches with $6 \mathrm{~dB}$ beamwidth of approximately $110^{\circ}$ in both the vertical and horizontal planes. All antennas were calibrated in an anechoic chamber. The gain of each antenna element is $6 \mathrm{dBi}$, with an angle resolution of $2^{\circ}$. This is limited by the sensors' distribution density in the anechoic chamber for antenna calibration. Powers received by the densely distributed sensors indicate the antenna power gain in the corresponding direction of the sensors, both in azimuth and in elevation domain. Angle information for paths can be extracted by substituting all possible angle values inside the antenna calibration file iteratively until the reconstructed signal best fits the actual received one [27]. The practical received signal will be deeply affected by the equipment's performance as it is the convolutional result of the measurement equipment impulse response (EIR) and CIR. To eliminate the potential influences of equipment performance, the equipment will be also calibrated with the $\mathrm{Tx}$ and $\mathrm{Rx}$ being connected directly through an ideal RF cable for the EIR file. By removing the EIR from the practical received signal, the CIR can be extracted. Table 1 specifies the configuration and angle range of the antenna arrays along with other measurement parameters. The angle ranges capture most of the propagation paths at both ends of the link, where paths with a delay interval larger than the delay resolution can be distinguished.

Utilizing both the horizontal and vertical dimensions, 3D MIMO is particularly suitable for scenarios with vertical user location distributions. Transmissions from outdoor BSs to users located at different floors can be well separated in their elevation angles.

We carried out the field measurements for 3D CIR in Beijing, China, and Auckland, New Zealand, for the O2I scenario. In China measurement, the receive antenna array was positioned at 5 different floors of a $60 \mathrm{~m}$ high building, while the transmit antenna was fixed at the top of a nearby $11.5 \mathrm{~m}$ high building at a distance of $21.5 \mathrm{~m}$. This typical O2I scenario is shown in Figure 2(a). On each floor, approximately 20 different fixed locations were measured, including ones near the window (the red star) or along the corridor, with 500 snapshots collected by the sounder for each location. Walls along the corridor and between the rooms are made of brick, 


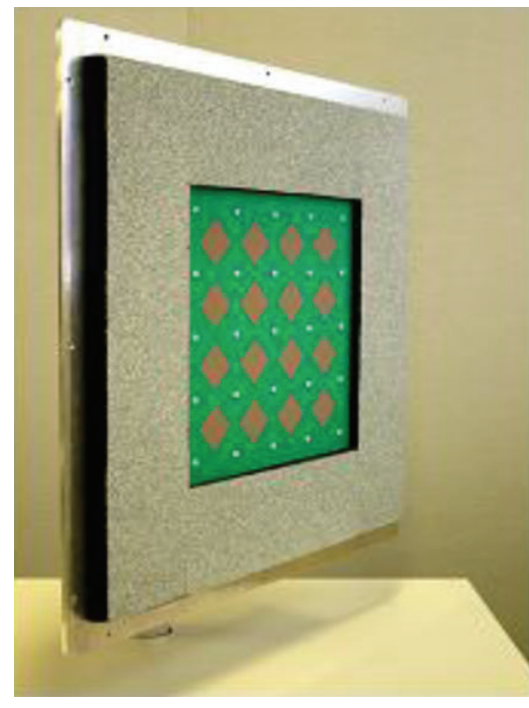

(a) UPA at Tx

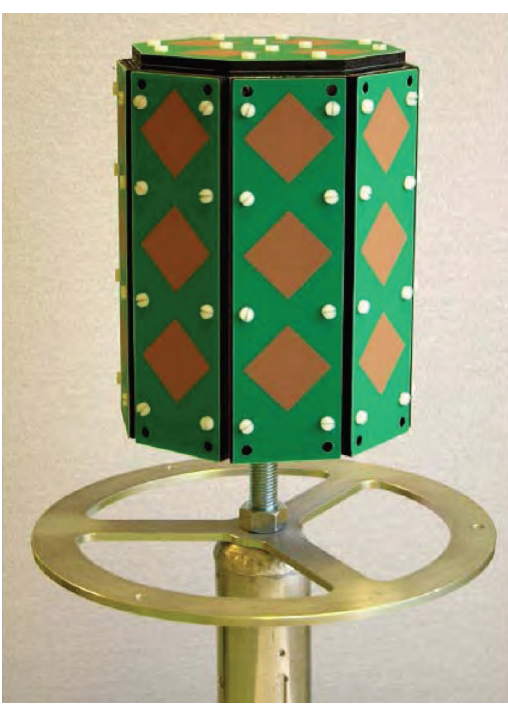

(b) ODA at Rx

Figure 1: Antenna layouts used in the measurements.

TABLE 1: Antenna configuration and parameters used in measurements.

\begin{tabular}{lcc}
\hline Parameter & \multicolumn{2}{c}{ Value } \\
\hline Antenna type & ODA & UPA \\
Element number & 56 & 32 \\
Element number used & 16 & 16 \\
Polarized & $\pm 45^{\circ}$ & $\pm 45^{\circ}$ \\
Distribution of antenna elements & Cylinder & Planar \\
Angle range & & \\
$\quad$ Azimuth & {$\left[-180^{\circ}, 180^{\circ}\right]$} & {$\left[-70^{\circ}, 70^{\circ}\right]$} \\
\multicolumn{1}{c}{ Elevation } & {$\left[-70^{\circ}, 90^{\circ}\right]$} & {$\left[-70^{\circ}, 70^{\circ}\right]$} \\
Carrier & $3.5 \mathrm{GHz}$ (China), $2.35 \mathrm{GHz}(\mathrm{NZ})$ \\
Bandwidth & $100 \mathrm{MHz}$ (China), $10 \mathrm{MHz}(\mathrm{NZ})$ \\
Tx power & $33 \mathrm{dBm}$ (China), $26 \mathrm{dBm}(\mathrm{NZ})$ \\
PN sequence & \multicolumn{2}{c}{63 (China), $31(\mathrm{NZ})$} \\
\hline
\end{tabular}

with plasterboard on the surface. The floor is covered with marble, while the doors of the rooms are wooden. The Rx was fixed on a trolley with an antenna height of $1.8 \mathrm{~m}$. With the exception of the first level, all floors featured a similar layout. Care was taken to ensure identical measurement locations on all floors.

The NZ measurement, shown in Figure 2(b), was performed at a cell site with the Tx antenna installed on the rooftop of a $45 \mathrm{~m}$ high building with its boresight pointing to the measurement building which is approximately $100 \mathrm{~m}$ far away. The room size is $30 \mathrm{~m}, 25 \mathrm{~m}$, and $4 \mathrm{~m}$ in length, width, and height, respectively. Approximately 80 fixed locations in the same floor were measured, with 200 snapshots collected for each location.

2.2. Data Postprocessing for 3D Channel Impulse Response. The field measurements described above provided numerous snapshots of the impulse response of the time-varying radio channel. The collected channel impulse responses were fed to a high-resolution algorithm to estimate the channel parameters for each snapshot. Maximum likelihood estimation (MLE) provides an optimum unbiased estimation from a statistical perspective; however, it is computationally prohibitive due to the multidimensional searches required. Thus a low-complexity approximation of MLE, spatialalternating generalized expectation-maximization (SAGE) algorithm [27], has been proposed to extract the key channel parameters on the basis of the newly released 3D channel model [17]. Denoting the total number of clusters by $C$, we have the following parameters for the $l$ th subpath in the $c$ th cluster:

(1) complex polarization (vertical $(V)$ ) and (horizontal $(H))$ components $\left[\alpha_{c, l}^{V V}, \alpha_{c, l}^{V H}, \alpha_{c, l}^{H V}, \alpha_{c, l}^{H H}\right]$,

(2) delays $\tau_{c, l}$,

(3) azimuth angle-of-departure (AoD) $\phi_{c, l}$ and angle-of$\operatorname{arrival}(\mathrm{AoA}) \varphi_{c, l}$,

(4) elevation AoD (EAoD) $\theta_{c, l}$ and AoA (EAoA) $\vartheta_{c, l}$,

(5) Doppler frequency $f_{d c, l}$ being 0 for fixed spots.

A single link of the 3D fading channel, between the base station and the mobile user, is shown in Figure 3. With $M$ number of transmits and $N$ number of receive antennas, for the cluster $c$, the 3D channel model is presented:

$$
\begin{aligned}
\mathbf{h}_{n, m, c}(t)= & \sum_{l=1}^{L}\left[\begin{array}{l}
F_{n, V} \\
F_{n, H}
\end{array}\right]^{T}\left[\begin{array}{ll}
\alpha_{c, l}^{V V} & \alpha_{c, l}^{V H} \\
\alpha_{c, l}^{H V} & \alpha_{c, l}^{H H}
\end{array}\right]\left[\begin{array}{l}
F_{m, V} \\
F_{m, H}
\end{array}\right] \\
& \cdot \exp \left(j 2 \pi \lambda_{0}^{-1}\left(\widehat{r}_{n, c, l}^{T} \cdot \bar{d}_{n}\right)\right) \\
& \cdot \exp \left(j 2 \pi \lambda_{0}^{-1}\left(\widehat{r}_{m, c, l}^{T} \cdot \bar{d}_{m}\right)\right),
\end{aligned}
$$



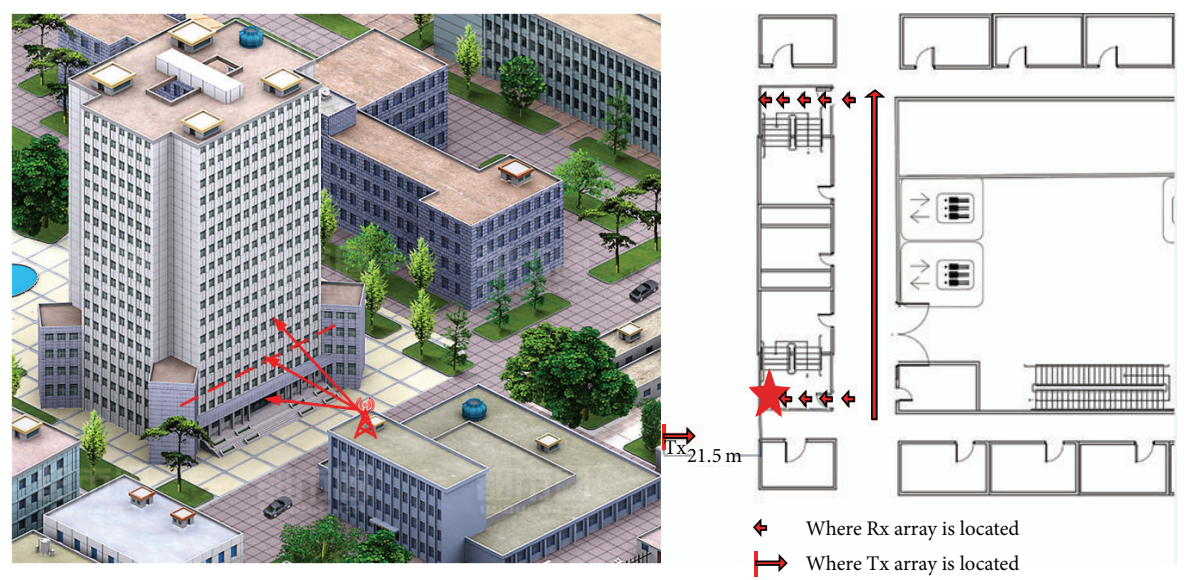

(a) Measurement in China
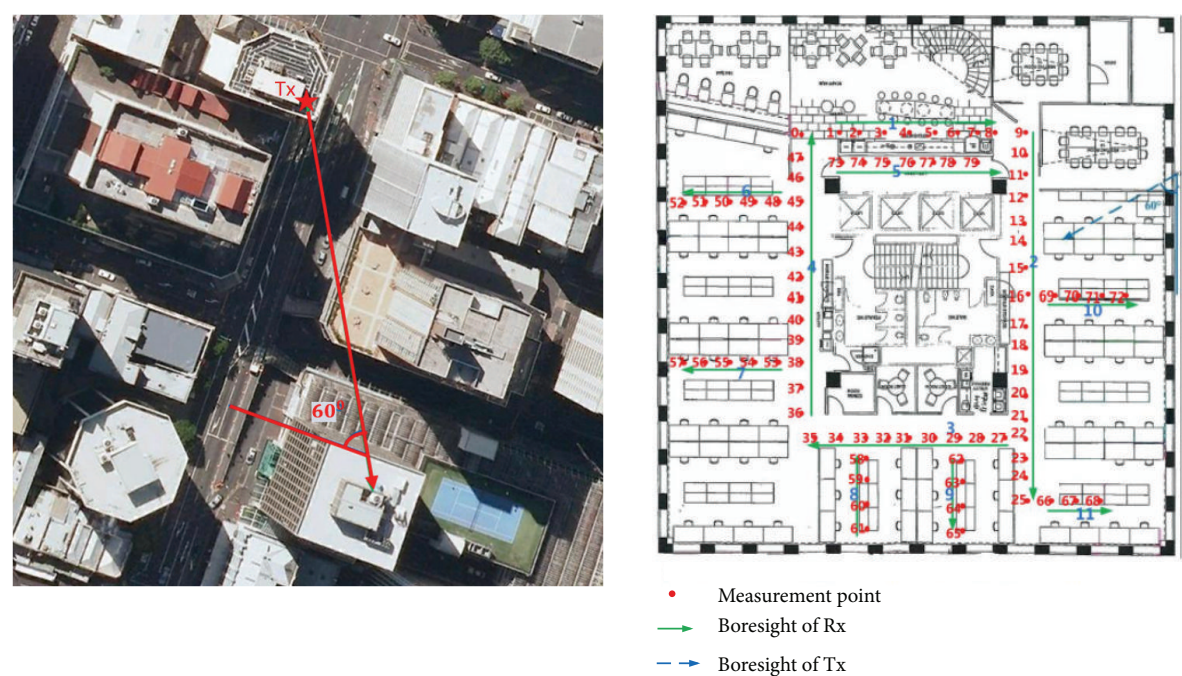

(b) Measurement in NZ

FIgURE 2: A view of measurement areas in China (a) and NZ (b).

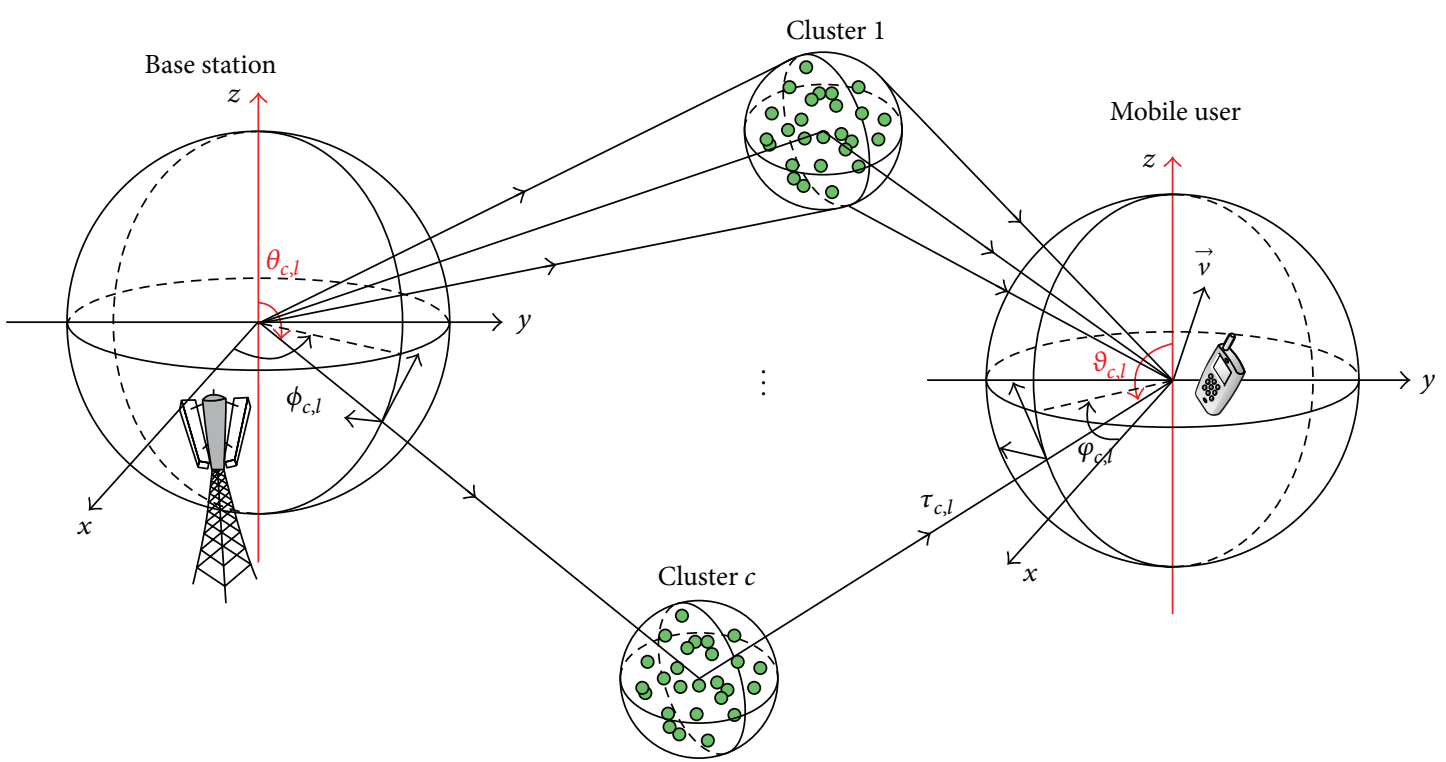

FIGURE 3: Generic 3D fading channel model. 


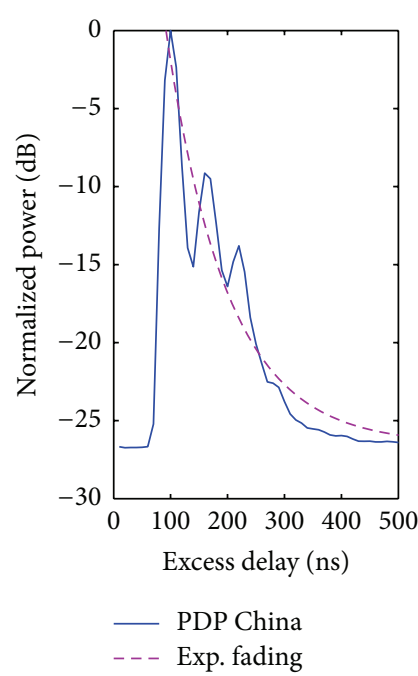

(a) Average PDP of measurements in China and NZ

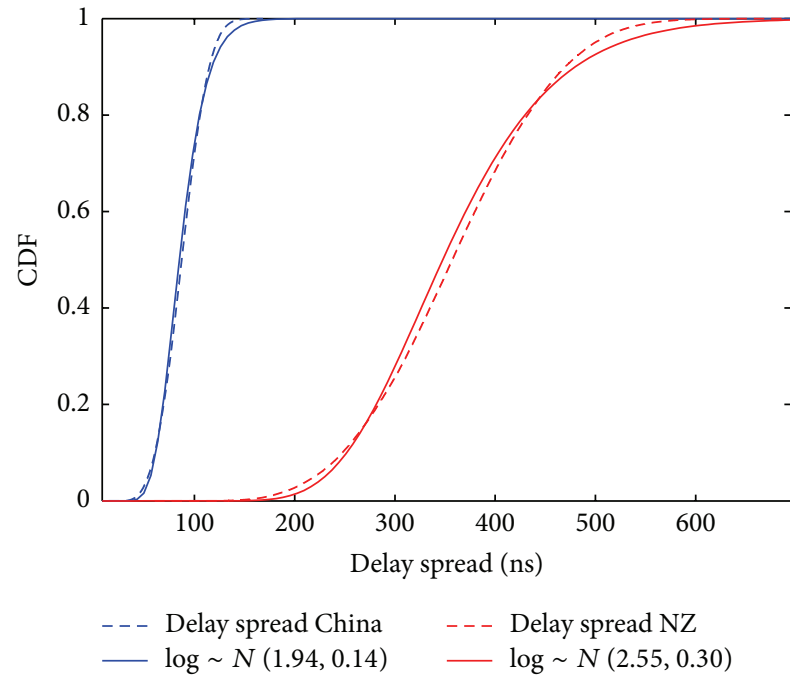

(b) RMS delay spread in China and NZ

FIgURE 4: PDP and delay spread in China and NZ.

where $L$ is the total number of subpaths, $\lambda_{0}$ is the wavelength of the carrier frequency, $n=1 \cdots N$, and $m=1 \cdots M \cdot \bar{d}_{m}$ and $\bar{d}_{n}$ are the 3D location vectors for Tx and Rx antennas, respectively. $\widehat{r}_{m, c, l}$ and $\hat{r}_{n, c, l}$ denote the spherical unit vectors at transmitter and receiver elements, respectively. For the $l$ th subpath in the $c$ th cluster, $\widehat{r}_{m, c, l}$ is calculated by

$$
\widehat{r}_{m, c, l}=\left[\begin{array}{c}
\sin \left(\theta_{c, l}\right) \cos \left(\phi_{c, l}\right) \\
\sin \left(\theta_{c, l}\right) \sin \left(\phi_{c, l}\right) \\
\cos \left(\theta_{c, l}\right)
\end{array}\right] .
$$

A similar expression is used to obtain spherical unit vector $\widehat{r}_{n, c, l}$ for Rx by substituting angles $\vartheta_{c, l}$ and $\varphi_{c, l}$ into (2). Besides, $F_{m, V}$ and $F_{m, H}$ are transmit antenna field patterns for the vertical and horizontal polarizations, respectively. Meanwhile, $F_{n, V}$ and $F_{n, H}$ are antenna field patterns for the vertical and horizontal polarizations on the Rx side.

\section{Results of Measurements}

The distribution results along with the 3GPP reference value are summarized in Table 2; the previous extracted parameters, both for China and for NZ measurements, are fed back to a further statistical characteristic analysis, including delay spread, angle distribution, and XPD.

3.1. Power Delay Profile and Delay Spread. Power delay profiles (PDP), for both China and NZ measurements, are presented for an intuitive glance over multipaths, dynamic power range and power fading with delays, and so forth. With a bandwidth of being $100 \mathrm{MHz}$ for China and $10 \mathrm{MHz}$ for $\mathrm{NZ}$ measurement, the delay resolution is $10 \mathrm{~ns}$ and $100 \mathrm{~ns}$, respectively. Based on the CIR exactly, the averaged PDP over all snapshots is shown in Figure 4(a). The peak value of the PDP has been normalized to $0 \mathrm{~dB}$. As a result of
TABLE 2: MIMO channel model parameters.

\begin{tabular}{|c|c|c|c|}
\hline Parameters & China & $\mathrm{NZ}$ & 3GPP [17] \\
\hline \multicolumn{4}{|l|}{ RMS delay, $\tau$, spread $\log _{10}(\mathrm{~s})$} \\
\hline$\mu$ & -7.06 & -6.45 & -6.62 \\
\hline$\sigma$ & 0.14 & 0.30 & 0.32 \\
\hline \multicolumn{4}{|l|}{ Elevation AoD, $\theta$, spread $\log _{10}$ (degrees) } \\
\hline$\mu$ & 1.28 & 1.13 & - \\
\hline$\sigma$ & 0.22 & 0.28 & - \\
\hline \multicolumn{4}{|l|}{ Elevation AoA, $\vartheta$, spread $\log _{10}$ (degrees) } \\
\hline$\mu$ & 1.39 & 1.56 & 1.01 \\
\hline$\sigma$ & 0.31 & 0.24 & 0.43 \\
\hline \multicolumn{4}{|l|}{ Azimuth AoD, $\phi$, spread $\log _{10}$ (degrees) } \\
\hline$\mu$ & 1.09 & 1.25 & 1.25 \\
\hline$\sigma$ & 0.14 & 0.16 & 0.42 \\
\hline \multicolumn{4}{|l|}{ Azimuth AoA, $\varphi$, spread $\log _{10}$ (degrees) } \\
\hline$\mu$ & 1.76 & 1.92 & 1.76 \\
\hline$\sigma$ & 0.10 & 0.11 & 0.16 \\
\hline \multicolumn{4}{|c|}{ Cross-polarization discrimination (XPD) } \\
\hline \multicolumn{4}{|l|}{$\mathrm{XPD}=10 \log _{10}\left(\left|\begin{array}{l}\alpha^{V V}+\alpha^{H H} \\
\alpha^{H V}+\alpha^{V H}\end{array}\right|^{2}\right)(\mathrm{dB})$} \\
\hline$\mu$ & 4.5 & 5.2 & 9 \\
\hline$\sigma$ & 6.4 & 9.1 & 11 \\
\hline Delay distribution & & EX & \\
\hline Azimuth AoA and AoD distribution & & Gauss & $\operatorname{sian}$ \\
\hline Elevation AoA and AoD distribution & & Lapla & cian \\
\hline
\end{tabular}

the complicated practical scattering environment, there exist three peaks both for China and for NZ measurements; then the PDP comes to a flat fading gradually. The noise floor, which may result from the system thermal noise and channel 


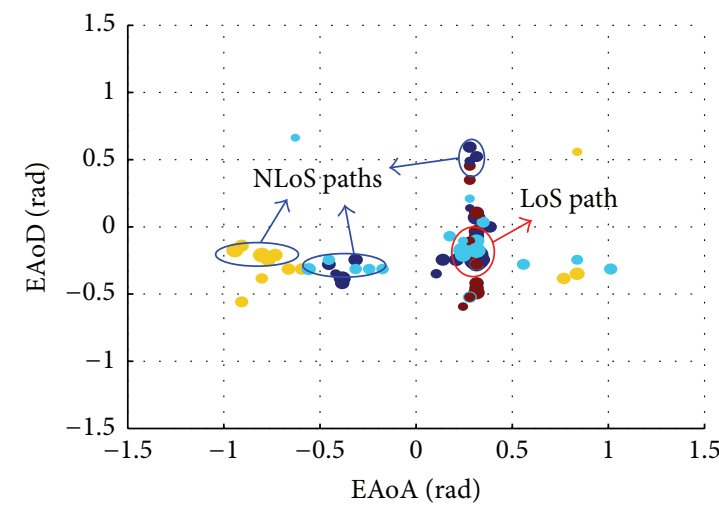

(a) EAoA versus EAoD at Floor 1

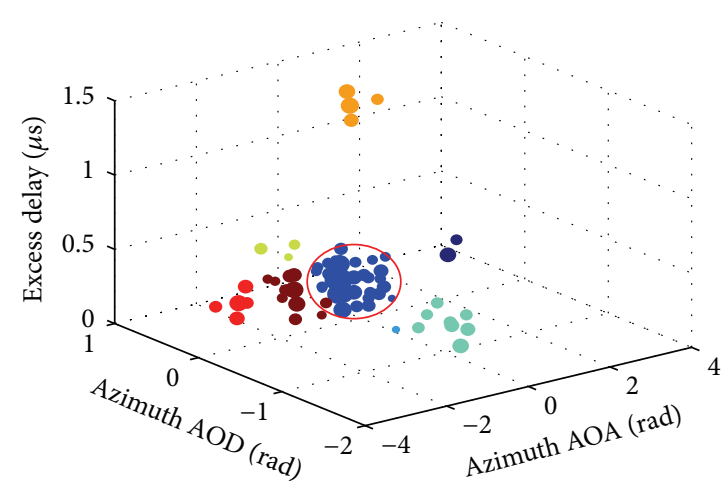

(c) 2 D clustering example

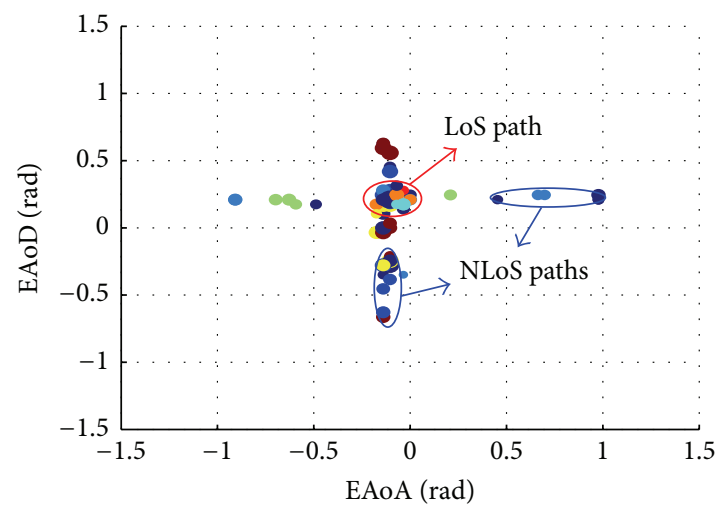

(b) EAoA vversus EAoD at Floor 3

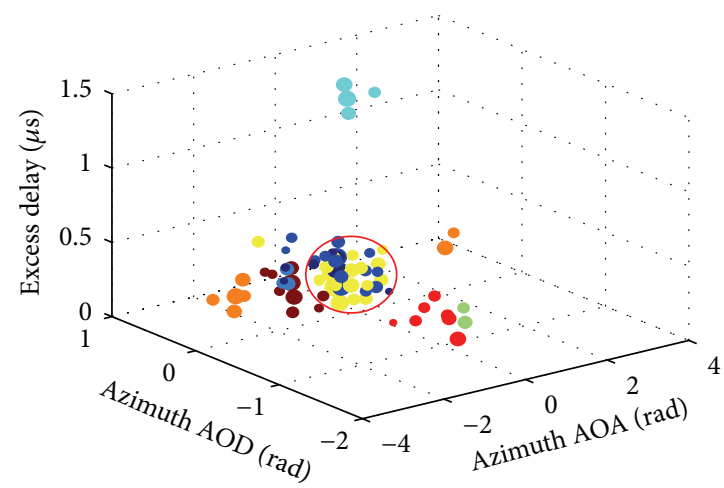

(d) $3 \mathrm{D}$ clustering example

Figure 5: EAoA and EAoD dispersion and 2D versus 3D clustering for the snapshot near the window (red star) in China measurement.

background noise, is calculated as the mean power of the relatively flat fading part of the PDP curve. The dynamic range between the peak power and bottom noise is $25 \mathrm{~dB}$ for China measurement and $20 \mathrm{~dB}$ for NZ measurement, which is $5 \mathrm{~dB}$ smaller because of a longer propagation distance; thus the peak value experienced a deeper fading. Power of MPCs after the reference delay of the strongest power decays rapidly in an exponential formation, which is the same with the conventional 2D PDP fading distribution [28], thus signal propagation in $3 \mathrm{D}$ environment has no obvious impact on the PDP.

For each snapshot, we also calculate the delay spread and present the cumulative distribution function $(\mathrm{CDF})$ of all $\mathrm{rms}$ DS in Figure 4(b) for both China and NZ measurements. There is an obvious lognormal distribution with related mean values and standard deviations presented in Table 2. Compared to China measurement, the DS in NZ is much larger because the room size of Rx is much larger in NZ measurement with all spots fixed in a wild area in the same floor.

3.2. EAoA and EAoD Dispersion and Clustering. The EAoA and EAoD dispersion at different floors are presented for China measurement. Taking the 1st and 3rd floor for example, the dispersions of EAoA-EAoD for the snapshot near the window (red star in Figure 2(a)) are shown in Figures 5(a) and
TABLE 3: Cluster number increase from $2 \mathrm{D}$ to $3 \mathrm{D}$.

\begin{tabular}{lccccc}
\hline Floor & 1st & 2nd & 3rd & 5th & 7th \\
\hline CluNum 2D & 10.2 & 8.4 & 9.3 & 9.5 & 12.2 \\
CluNum 3D & 15.2 & 12.5 & 13.4 & 15.4 & 17.4 \\
\hline
\end{tabular}

5(b), respectively. Points in the same color are divided into the same cluster while the size represents the path power. There is a brief marker of the LoS path which comes through windows directly while the NLoS path might be reflected by concrete walls. Given a height of $14 \mathrm{~m}$ at Tx which is slightly lower than $\mathrm{Rx}$ at the 3rd floor, the EAoD of the LoS path, being slightly bigger than 0 degree, can be expected as shown in Figure 5(b).

As multipath components (MPCs) are dispersive for both 1st and 3rd floor in the 3D environment, an interesting question arises whether the cluster number will change compared to that in 2D channel? To identify clusters [29] for the same snapshot near the window for the 3rd floor, a comparative result of the estimated MPCs for China measurements is presented in Figures 5(c) and 5(d). Cluster number increases from 7 to 9 , the main cluster in blue for 2D case divides into 2 subclusters for 3D case. An averaged cluster number for all snapshots on each floor is also presented in Table 3; the cluster increase from $2 \mathrm{D}$ to $3 \mathrm{D}$ indicates that a more specific channel propagation characteristic can be observed in a $3 \mathrm{D}$ vision. 

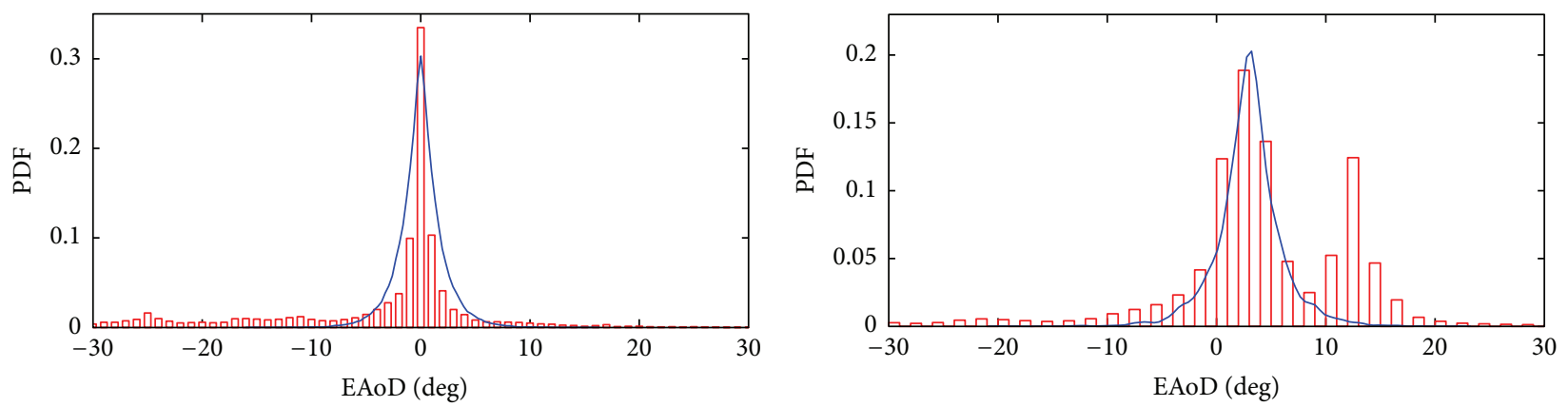

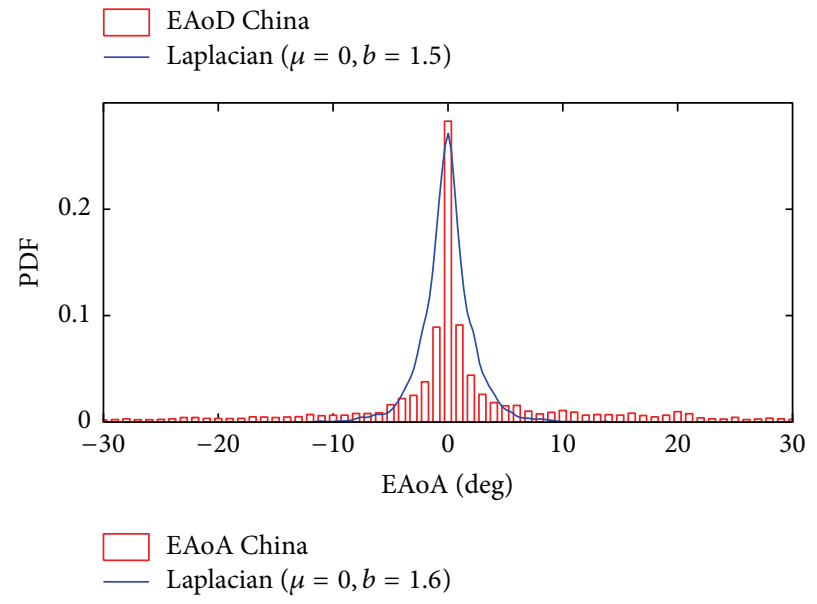

(a) EAoD and EAoA distribution in China measurement

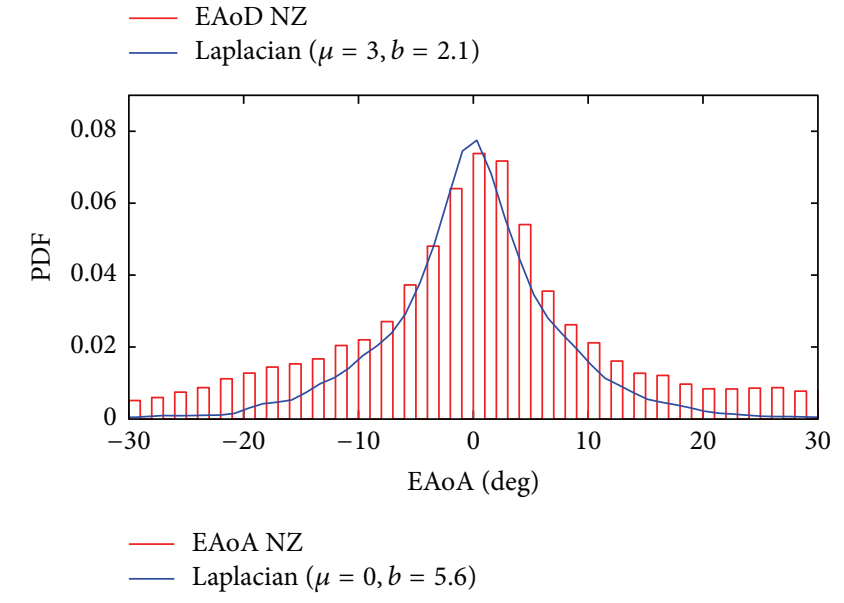

(b) EAoD and EAoA distribution in NZ measurement

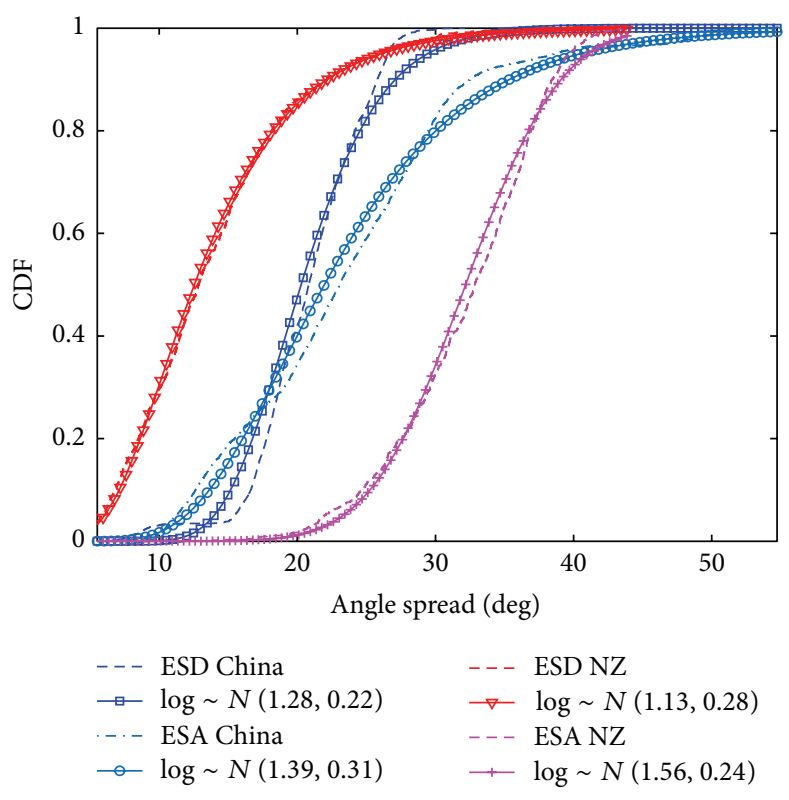

(c) EASD and EASA CDFs in NZ

FIgURE 6: EAoA and EAoD distributions and angular spread.

3.3. EAoA and EAoD Distributions and Angular Spread. The distribution of EAoD and EAoA for China and NZ measurements is shown in Figures 6(a) and 6(b), respectively. With the EAoD and EAoA of the strongest path both being shifted to zero degrees for all measured spots, figures show that the angle in China measurement exhibits a well Laplacian fit, as presented by

$$
f(x)=\frac{1}{\sqrt{2} \sigma} \exp \left(-\frac{\sqrt{2}}{\sigma}|x-\mu|\right),
$$




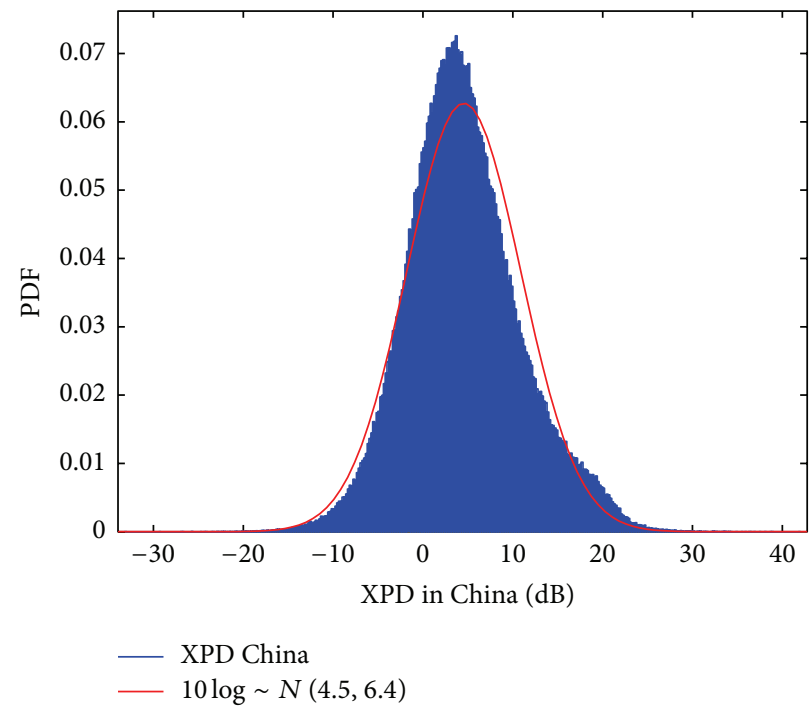

(a) PDF of XPD in China

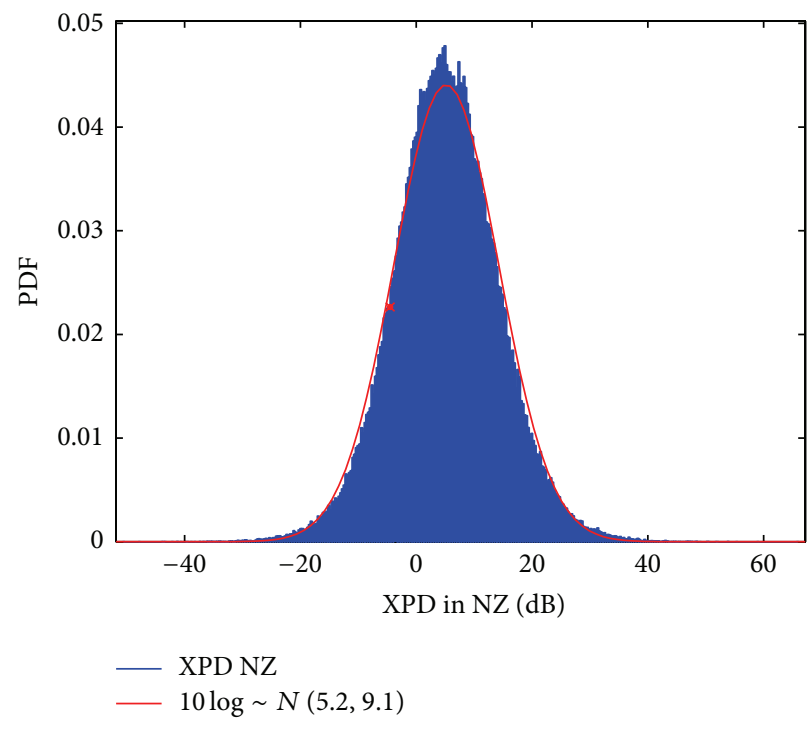

(b) PDF of XPD in NZ

FIGURE 7: XPD measurements for China and NZ.

where $\mu$ and $\sigma^{2}$ are the mean value and variance of the Laplace distribution, respectively. However, this is not the case for NZ result. A possible reason for this is the lack of sample data for only one floor measurement. In addition to the elevation power spectrum mentioned above, rms angle spread provides another perspective for a further understanding of 3D MIMO channel. For each snapshot, the angular spread in the elevation domain can be calculated. Taking all the subpaths in the $c$ th cluster for example, the angular spread of EAoD is given by

$$
\sigma_{\mathrm{AS}}=\sqrt{\frac{\sum_{l=1}^{L}\left(\theta_{c, l}-\left[\sum_{l=1}^{L} \theta_{c, l} P_{c, l} / \sum_{l=1}^{L} P_{c, l}\right]\right)^{2} P_{c, l}}{\sum_{l=1}^{L} P_{c, l}}},
$$

where the power of the $l$ th subpath in the $c$ th cluster can be calculated as the sum of squares of the polarization coefficients, given by

$$
P_{c, l}=\left|\alpha_{c, l}^{V V}\right|^{2}+\left|\alpha_{c, l}^{V H}\right|^{2}+\left|\alpha_{c, l}^{H V}\right|^{2}+\left|\alpha_{c, l}^{H H}\right|^{2} .
$$

The CDF of elevation angular spread for AoD (EASD) and AoA (EASA) is shown in Figure 6(c), for both China and NZ measurements. Similar to 3GPP [17], a lognormal distribution fits well with this angular spread with the mean and standard deviation presented in Table 2. As we can see, EASD is smaller than EASA due to an additional channel propagation and scattering for transmitted signals. Besides, the AS of elevation angles are correspondingly smaller than that of azimuth angles, both for departure and for arrival sides. It is reasonable that vertical scatters are not so rich compared to the azimuth plane. What is more, the ESA in NZ is larger than that of China while the ESD is smaller; this can be explained by a much larger room size with complex scatters on the Rx side in NZ measurement. Meanwhile, on Tx
TABLE 4: Angular spread for different floors in China measurement.

\begin{tabular}{lcccc}
\hline Floor & EASD $\left(^{\circ}\right)$ & EASA $\left(^{\circ}\right)$ & AASD $\left(^{\circ}\right)$ & AASA $\left(^{\circ}\right)$ \\
\hline 1st & 20.8 & 27.4 & 20.4 & 45.5 \\
2nd & 20.6 & 20.9 & 12.5 & 42.2 \\
3rd & 19.5 & 22.3 & 19.3 & 43.3 \\
5th & 20.8 & 22.1 & 18.0 & 40.9 \\
7th & 26.0 & 28.3 & 17.9 & 43.1 \\
\hline
\end{tabular}

side, so long the propagation distance is that only paths in a specific EoD can be received in NZ measurement, which leads to a concentrated ESD. A more specific angular spread for different floors in China measurement is presented in Table 4.

3.4. XPD Distribution. As XPD is being defined in Table 2, its distribution is shown in Figure 7 for both China and NZ measurements. Similar to 3GPP, a normal distribution fits XPD well for all fixed spots. In NZ result, the range of XPD is wider than that of China as the room size on $\mathrm{Rx}$ side in $\mathrm{NZ}$ is much larger, which will result in a more complex depolarization in $3 \mathrm{D}$ propagation. As XPD is closely related to the environment, the mean value of XPD for different floors varies in China measurement, being 3.3, 5.1, 5.3, 3.8, and 3.1 for the 1st, 2nd, 3rd, 5th, and 7th floor, respectively. To be noted, the Tx is as much high as Rx on the 3rd floor; a higher distance in elevation domain will contribute to a better vertical depolarization.

\section{Conclusion}

In this paper, we studied the statistical characteristics of 3D channel impulse response and presented comparative results for measurements performed in China and NZ. We have focused our study on the elevation domain as these 
measurements are rare. Simulation results show that the PDP in $3 \mathrm{D}$ propagation fades exponentially both in China and in NZ measurements. The cluster can be further resolved when the elevation domain is taken into consideration. A lognormal distribution fits well with the AS while the ESA in $\mathrm{NZ}$ is larger than that of China due to a larger scattering room size in NZ measurement. Because of a more dispersed distribution of measured locations in the elevation domain, the XPD in China is smaller than that of NZ and the XPD varies with the height difference between $\mathrm{Tx}$ and $\mathrm{Rx}$, and so forth. All these results of the elevation domain provide a better understanding of $3 \mathrm{D}$ channel and contribute to 3D MIMO system design, including 3D antenna arrays for future $5 \mathrm{G}$ where the elevation domain cannot be ignored. Therefore, these measurements are a precursor to $5 \mathrm{G}$ systems capability studies.

\section{Conflict of Interests}

The authors declare that there is no conflict of interests regarding the publication of this paper.

\section{Acknowledgments}

The China measurements are supported in part by the 863 Program of China under Grant no. 2015AA01A703 and by National Science and Technology Major Project of the Ministry of Science and Technology (project name is "IMT-2002 Candidate Band Analysis and Evaluation") with 2015ZX03002008 and by National Science and Technology Major Project of the Ministry of Science and Technology (project name is "Wireless mobile spectrum research and verification for WRC15”) with 2014ZX03003013-004 and by National Science and Technology Major Project of the Ministry of Science and Technology (project name is "Research and Development of MIMO Channel Emulator") with 2013ZX03001020-002 and by Huawei Company for Massive MIMO study.

\section{References}

[1] Cisco, "Cisco visual networking index: global mobile data traffic forecast update, 2013-2018," Cisco White Paper, Cisco, 2014.

[2] J. G. Andrews, S. Buzzi, W. Choi et al., "What will 5G be?" IEEE Journal on Selected Areas in Communications, vol. 32, no. 6, pp. 1065-1082, 2014.

[3] X. Cheng, B. Yu, L. Yang et al., "Communicating in the real world: 3D MIMO," IEEE Wireless Communications, vol. 21, no. 4, pp. 136-144, 2014.

[4] M. Shafi, M. Zhang, A. L. Moustakas et al., "Polarized MIMO channels in 3-D: models, measurements and mutual information," IEEE Journal on Selected Areas in Communications, vol. 24, no. 3, pp. 514-526, 2006.

[5] T. Aulin, "A modified model for the fading signal at a mobile radio channel," IEEE Transactions on Vehicular Technology, vol. 28, no. 3, pp. 182-203, 1979.

[6] M. Shafi, M. Zhang, P. J. Smith, A. L. Moustakas, and A. F. Molisch, "The impact of elevation angle on MIMO capacity," in Proceedings of the IEEE International Conference on Communications (ICC '06), pp. 4155-4160, Istanbul, Turkey, July 2006.
[7] O. N. C. Yilmaz, S. Hämäläinen, and J. Hämäläinen, "System level analysis of vertical sectorization for 3GPP LTE," in Proceedings of the 6th International Symposium on Wireless Communication Systems (ISWCS '09), pp. 453-457, IEEE, Tuscany, Italy, September 2009.

[8] Y.-H. Nam, B. L. Ng, K. Sayana et al., "Full-dimension MIMO (FD-MIMO) for next generation cellular technology," IEEE Communications Magazine, vol. 51, no. 6, pp. 172-179, 2013.

[9] C. Pan and J. Zhang, "Experimental investigation of elevation angles and impacts on channel capacity in urban microcell," in Proceedings of the International Conference on Computing, Networking and Communications (ICNC '15), pp. 11-15, IEEE, Garden Grove, Calif, USA, Feburary 2015.

[10] K. Kalliola, H. Laitinen, L. I. Vaskelainen, and P. Vainikainen, "Real-time 3-D spatial-temporal dual-polarized measurement of wideband radio channel at mobile station," IEEE Transactions on Instrumentation and Measurement, vol. 49, no. 2, pp. 439448, 2000.

[11] K. Kalliola, K. Sulonen, H. Laitinen, O. Kivekäs, J. Krogerus, and P. Vainikainen, "Angular power distribution and mean effective gain of mobile antenna in different propagation environments," IEEE Transactions on Vehicular Technology, vol. 51, no. 5, pp. 823-838, 2002.

[12] K. Kalliola, H. Laitinen, P. Vainikainen, M. Toeltsch, J. Laurila, and E. Bonek, "3-D double-directional radio channel characterization for urban macrocellular applications," IEEE Transactions on Antennas and Propagation, vol. 51, no. 11, pp. 3122-3133, 2003.

[13] J. Medbo, M. Riback, H. Asplund, and J.-E. Berg, "Mimo channel characteristics in a small macrocell measured at $5.25 \mathrm{GHz}$ and $200 \mathrm{MHz}$ bandwidth," in Proceedings of the IEEE 62nd Vehicular Technology Conference (VTC-Fall '05), vol. 1, pp. 372376, IEEE, May 2005.

[14] L. Materum, J.-I. Takada, I. Ida, and Y. Oishi, "Mobile station spatio-temporal multipath clustering of an estimated wideband MIMO double-directional channel of a small urban $4.5 \mathrm{GHz}$ macrocell," EURASIP Journal on Wireless Communications and Networking, vol. 2009, Article ID 804021, p. 9, 2009.

[15] Z. Zhong, X. Yin, X. Li, and X. Li, "Extension of ITU IMT-A channel models for elevation domains and line-of-sight scenarios," http://arxiv.org/abs/1301.2518.

[16] M. Jiang, M. Hosseinian, M.-I. Lee, and J. Stern-Berkowitz, "3D channel model extensions and characteristics study for future wireless systems," in Proceedings of the IEEE 24th Annual International Symposium on Personal, Indoor, and Mobile Radio Communications (PIMRC '13), pp. 41-46, IEEE, London, UK, September 2013.

[17] 3GPP TR 36.873 V.2, "Study on 3D channel model for LTE (release 12)," Tech. Rep., 2014.

[18] J. Meinila, P. Kyosti, L. Hentila et al., "D5.3: WINNER+ final channel models V.1," in Proceedings of the Wireless World Initiative New Radio, 2010.

[19] J. Zhang, C. Pan, F. Pei, G. Liu, and X. Cheng, "Threedimensional fading channel models: a survey of elevation angle research," IEEE Communications Magazine, vol. 52, no. 6, pp. 218-226, 2014.

[20] R1-132543, "Uma channel measurements results on elevation related parameters," 3GPP TSG-RAN WG1 73, CMCC, CATT, Fukuoka, Japan, 2013.

[21] R1-133525, "UMi channel measurement results on elevation related parameters," in Proceedings of the 3GPP TSG-RAN WG1 74, CMCC, CATT, Barcelona, Spain, August 2013. 
[22] R1-132544, "O2i channel measurements results on elevation related parameters," 3GPP TSG-RAN WG1 74, CMCC, CATT, Barcelona, Spain, 2013.

[23] R1-134222, "Proposals for fast fading channel modelling for 3D UMi," 3GPP TSG-RAN WG1 74bis, Alcatel-Lucent Shanghai Bell, Alcatel-Lucent, China Unicom, Guangzhou, China, 2013.

[24] R1-134221, "Proposals for fast fading channel modelling for 3D UMa," in Proceedings of the 3GPP TSG-RAN WG1 74bis, Alcatel-Lucent Shanghai Bell, Alcatel-Lucent, China Unicom, Guangzhou, China, October 2013.

[25] R1-134795, "Proposals for fast fading channel modelling for 3D UMi O2I," in Proceedings of the 3GPP TSG-RAN WG1 74bis, Alcatel-Lucent Shanghai Bell, Alcatel-Lucent, China Unicom, Guangzhou, China, October 2013.

[26] J. Zhang, "Review of wideband MIMO channel measurement and modeling for IMT-Advanced systems," Chinese Science Bulletin, vol. 57, no. 19, pp. 2387-2400, 2012.

[27] B. H. Fleury, M. Tschudin, R. Heddergott, D. Dahlhaus, and K. I. Pedersen, "Channel parameter estimation in mobile radio environments using the SAGE algorithm," IEEE Journal on Selected Areas in Communications, vol. 17, no. 3, pp. 434-450, 1999.

[28] M. Series, "Guidelines for evaluation of radio interface technologies for IMT-Advanced," Report ITU 2135-1, 2009.

[29] N. Czink, P. Cera, J. Salo, E. Bonek, J.-P. Nuutinen, and J. Ylitalo, "A framework for automatic clustering of parametric MIMO channel data including path powers," in Proceedings of the IEEE 64th Vehicular Technology Conference (VTC-Fall '06), pp. 1-5, Montreal, Canada, September 2006. 


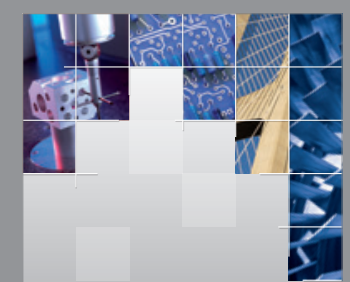

\section{Enfincering}
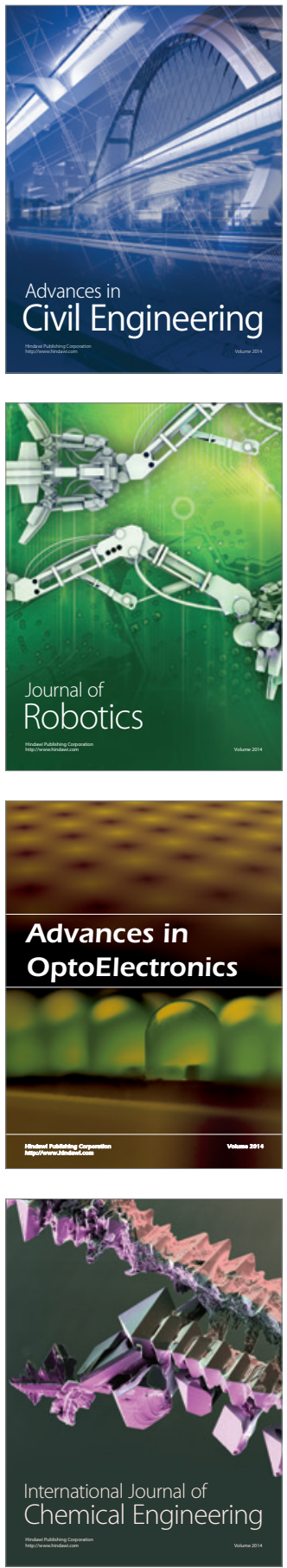

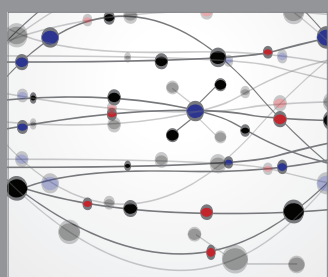

The Scientific World Journal

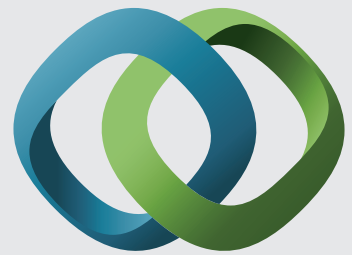

\section{Hindawi}

Submit your manuscripts at

http://www.hindawi.com
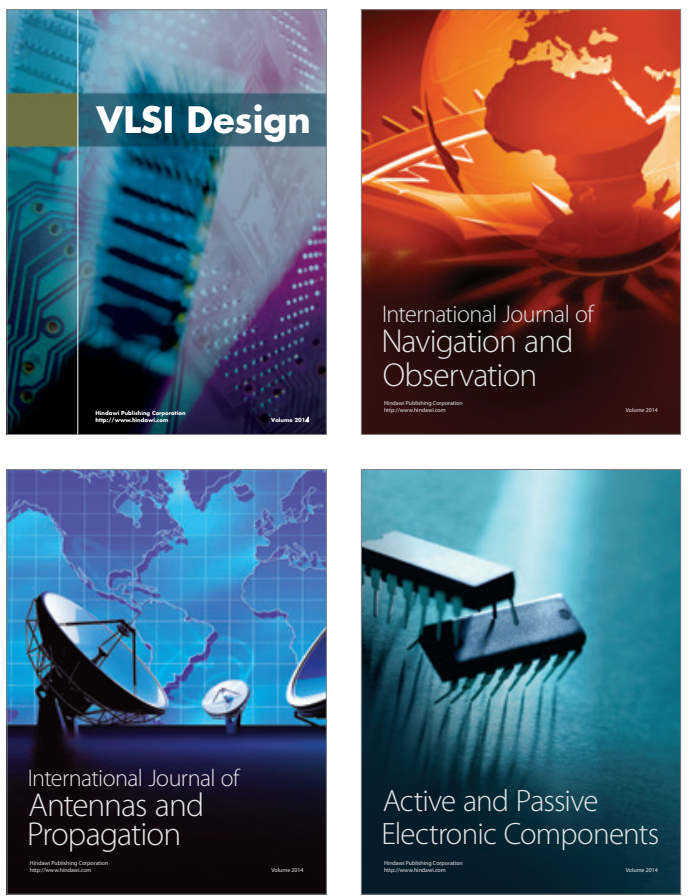
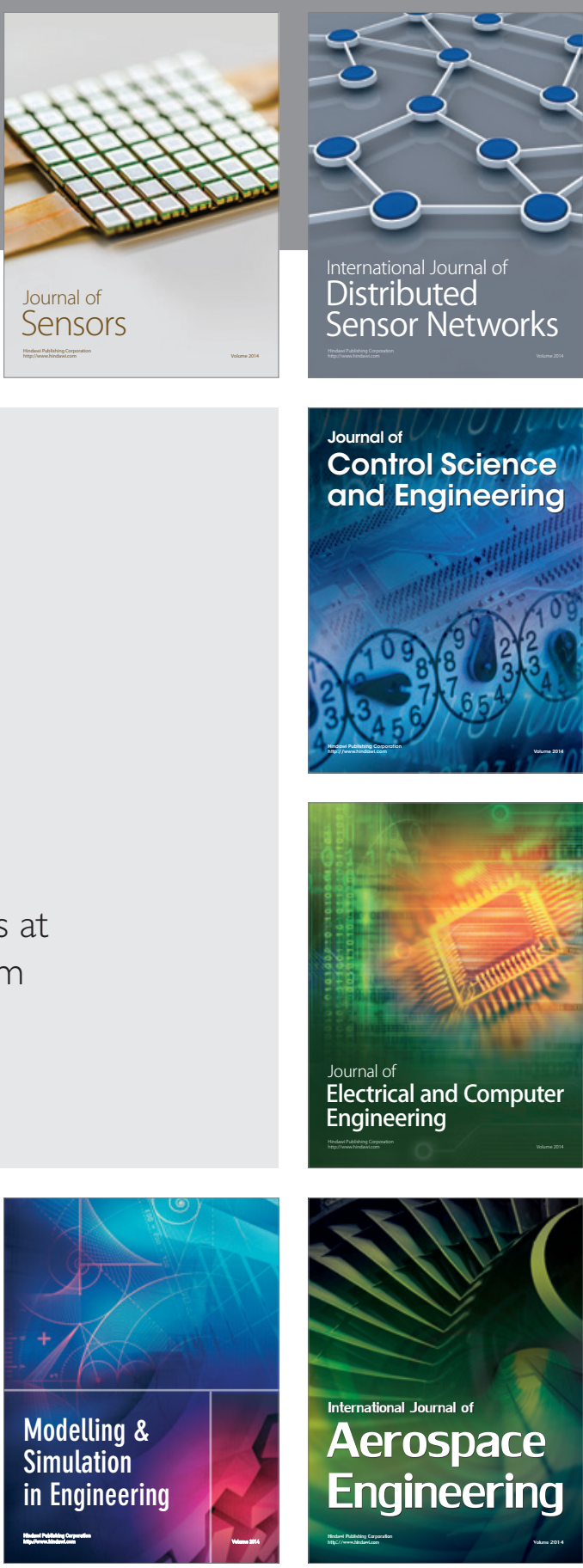

International Journal of

Distributed

Sensor Networks

Journal of

Control Science

and Engineering
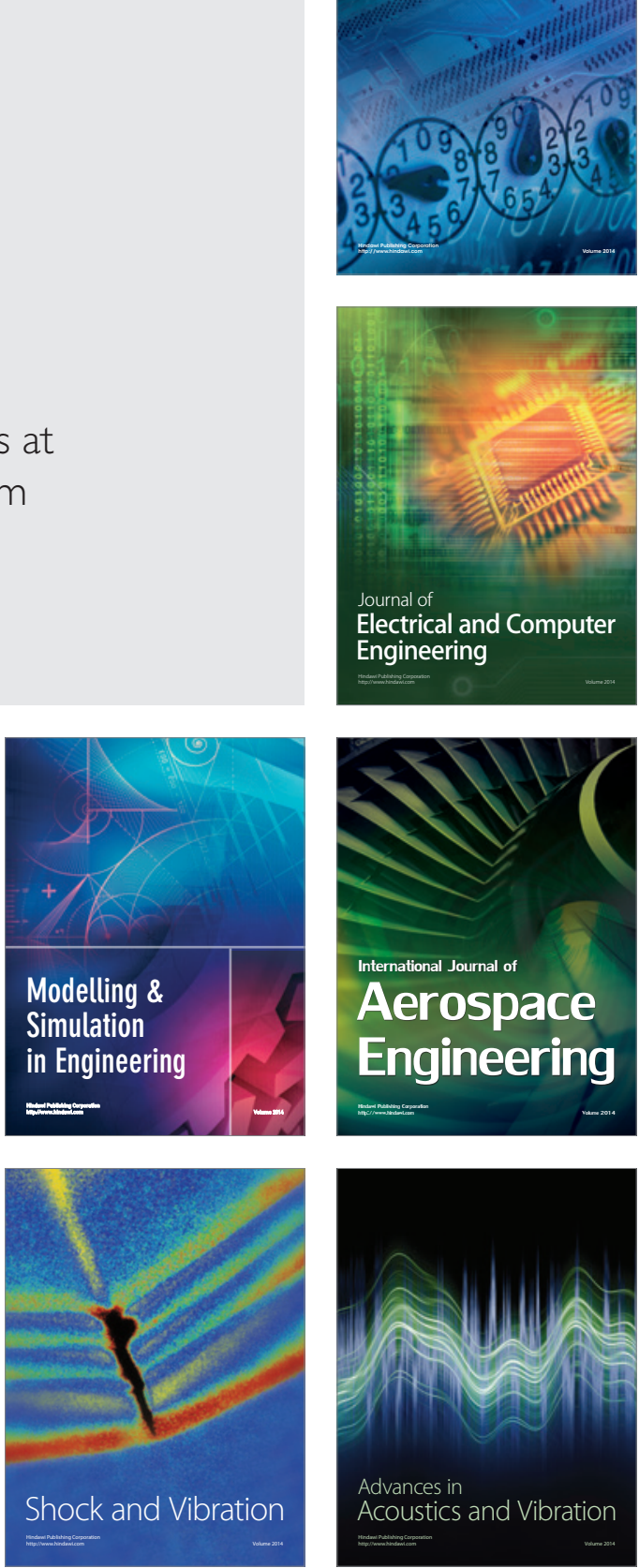